\title{
Evaluación de la capacidad de almacenamiento de energía en xerogeles de carbono activados obtenidos a partir de lignina
}

\author{
Storage energy capacity evaluation of \\ activated carbon xerogels from lignin
}

\section{Avaliação da capacidade de armazenamento de energia de xerogéis de carbono ativados a partir de lignina}

\author{
Giovanna Diossa ${ }^{1,2}$; Chris Daniela Castro ${ }^{2}$ Z Zulamita Zapata-Benabithe ${ }^{1 *}$; Germán Quintana ${ }^{2}$ \\ ${ }^{1}$ Grupo de Energía y Termodinámica, ${ }^{2}$ Grupo de Pulpa y Papel, Facultad de Ingeniería Química. Universidad \\ Pontificia Bolivariana, Circular 1 No 70-01, Medellín 050031, Colombia. \\ *zulamita.zapata@upb.edu.co
}

Recepción: 30 de noviembre de 2016

Fecha Aceptación: 15 de octubre de 2017

\begin{abstract}
Resumen
En este trabajo se prepararon dos tipos de xerogeles orgánicos a partir de soluciones sol-gel usando resorcinol $(R)$, formaldehido $(F)$, lignina $(L)$ y $\mathrm{NaOH}(C)$ como catalizador. La relación másica $L /(R+L)$ se fijó en 0,27 y el contenido de catalizador se varió en dos relaciones másicas de $(R+L) / C, 90,3$ (serie G) y 72,1 (serie D). Los xerogeles orgánicos se activaron químicamente con ácido fosfórico a una relación másica de ácido a xerogel de $0,85 / 1,1 \mathrm{~h}$ de impregnación y $2 \mathrm{~h}$ de activación y una temperatura de activación de $450^{\circ} \mathrm{C}$. Los xerogeles de carbono activados se evaluaron electroquímicamente como electrodos para supercondensadores mediante diferentes técnicas como voltamperometría cíclica (VC), cronopotenciometría (CP) y espectroscopía de impedancia electroquímica (EIS) en una configuración de dos y tres electrodos. El valor de la capacidad gravimétrica a partir de las curvas de voltamperometría cíclica a $0,5 \mathrm{mV} / \mathrm{s}(0-0,75 \mathrm{~V})$ para las muestras activadas GA27 y DA27 fueron 222 y $226 \mathrm{~F} / \mathrm{g}$, respectivamente. La muestra DA27 mostró una mayor retención de la energía, que es atribuible a un aumento de la presencia de mesoporos en la muestra comparada con la GA27. Los mesoporos se forman debido a la degradación de la lignina en la activación con ácido fosfórico; la muestra DA27 tiene una reticulación superior en comparación con la muestra GA27, ya que el interior del xerogel orgánico es menos accesible. La muestra con mayor contenido de catalizador mostró mejor comportamiento electroquímico para su aplicación como electrodos para supercondensadores.
\end{abstract}

Palabras clave: Lignina, Xerogeles de carbono activados, propiedades electroquímicas, Ácido Fosfórico.

\begin{abstract}
In this work two types of organic xerogels were prepared from sol-gel solutions using resorcinol $(R)$, formaldehyde $(F)$, lignin $(L)$ and $\mathrm{NaOH}(C)$ as catalyst. The mass ratio $L /(R+L)$ was set at 0.27 and the amount of catalyst was varied on two mass ratios ( $R+L) / C, 90.3$ ( $G$ series) and 72.1 (D series). Organic xerogels are chemically activated with phosphoric acid with a mass ratio of acid to xerogel $0.85 / 1,1 \mathrm{~h}$ of impregnation and $2 \mathrm{~h}$ of activation and activation temperature is fixed at $450^{\circ} \mathrm{C}$. Activated carbon xerogels are electrochemically evaluated as electrodes for supercapacitors using different techniques such as cyclic voltammetry (CV), chronopotentiometry (CP) and electrochemical impedance spectroscopy (EIS) in a configuration of two and three electrodes. The value of the gravimetric capacity from cyclic voltammetry curves at $0.5 \mathrm{mV} / \mathrm{s}(0-0.75 \mathrm{~V})$ for samples GA27 and DA27 were 222 and $226 \mathrm{~F} / \mathrm{g}$, respectively. The DA27 sample showed more retention of energy than GA27, which is attributable to an increase in the presence
\end{abstract}


of mesopores in the sample compared GA27. The mesopores are formed due to the degradation of lignin in activation with phosphoric acid; the DA 27 sample has more crosslinking than GA27 sample and this produces that inside the organic xerogel be less accessible. The sample with a higher content of catalyst showed better electrochemical behavior for use as electrodes for supercapacitors.

Keywords: Lignin, Activated carbon xerogels, electrochemical properties, Phosphoric acid.

\section{Resumo}

Neste trabalho foram preparados dois tipos de xerogéis orgânicos a partir de soluções de sol-gel utilizando o resorcinol $(R)$, formaldeído $(F)$, lignina $(L)$ e $\mathrm{NaOH}(C)$ como catalisador. A proporção de massa $L /(R+L)$ foi de 0,27 e o teor de catalisador foi variado em duas proporções de massa $(R+L) / C, 90,3$ (série de G) e 72,1 (série de D). Os xerogéis orgânicos são quimicamente ativados com o ácido fosfórico com uma relação de massa de ácido para o xerogel de $0,85 / 1$, tempos de impregnação e de ativação de uma hora e com uma temperatura de activação de $450^{\circ} \mathrm{C}$. Os xerogéis de carbono ativados foram avaliados eletroquimicamente como eletrodos para supercapacitores usando técnicas diferentes, tais como voltametria cíclica (CV), cronopotenciometria (PB) e espectroscopia de impedância eletroquímica (EIS) numa configuração de dois e três eletrodos. O valor da capacidade gravimétrica obtidas das curvas de voltametria cíclica para $0,5 \mathrm{mV} / \mathrm{s}(0-0,75 \mathrm{~V})$ para as amostras GA27 e DA27 foram de 222 e $226 \mathrm{~F} / \mathrm{g}$, respetivamente. A amostra DA27 apresentou maior retenção de energia, que é atribuível a um aumento da presença de mesoporos na amostra em comparação com a GA 27. Os mesoporos são formados devido à degradação da lignina na activação com ácido fosfórico; amostra DA27 tem uma maior reticulação comparada com a amostra GA27, já que o interior do xerogel orgânico é menos acessível. A amostra com maior teor de catalisador apresentou melhor desempenho eletroquímico para aplicação como eletrodos para supercapacitores.

Palavras-chave: Lignina, Xerogéis de carbono ativado, propriedades eletroquímicas, Ácido fosfórico.

\section{Introducción}

Los supercondensadores son dispositivos de almacenamiento de energía eléctrica reconocidos por almacenar energía eléctrica a altas densidades de potencia $\left(10-10^{6} \mathrm{~W} / \mathrm{kg}\right)$, tiempos de respuesta cortos $(0,3-3 \mathrm{~s})$ y alta estabilidad en cada ciclo de carga y descarga sin afectar su rendimiento [1,2]. Estos dispositivos son tecnológicamente atractivos en aplicaciones como equipos de telecomunicaciones, vehículos eléctricos/híbridos, fuentes de energía portátil, actuadores electroquímicos y en sistemas de generación de potencia con energía solar y eólica $[1,3,4]$. La carga eléctrica se almacena debido a la acumulación de iones en la superficie interna de ambos electrodos (ánodo-cátodo), los iones sin solvatar del electrolito se ajustan al poro formando una doble capa eléctrica en combinación con los electrones en el material conductor [5,6]. La capacidad de almacenamiento de energía en la doble capa se conoce como capacidad de la doble capa eléctrica y la capacidad debido a la presencia de reacciones de oxidación-reducción se conoce como pseudocapacidad [2].

Los electrodos de los supercondensadores son desarrollados a partir de materiales polarizables con una alta superficie, alta conductividad y poros adaptados al tamaño de los iones, con el propósito de mejorar el rendimiento de este tipo de dispositivos. Los más usados son materiales de carbono, óxidos metálicos y polímeros conductores [7]. Los materiales de carbono se utilizan comúnmente debido a su bajo costo, fácil disponibilidad, no tóxicos, ambientalmente amigables y estables $[8,10]$. Algunos de los materiales de carbono que se utilizan son carbones activados, geles de carbono, carbones mesoporosos ordenados, nanotubos de carbono, entre otros. En los materiales carbonosos los microporos entre 0,7 y $2 \mathrm{~nm}$ se caracterizan por un alto desempeño capacitivo, sin embargo la máxima capacidad de la doble capa es obtenida con una distribución de poros muy cercana al tamaño del ion del electrolito [11,12]. Los mesoporos son importantes debido a que son necesarios para el transporte eficiente de los iones [13]. Los poros muy grandes o pequeños pueden influenciar en una disminución de la capacidad [12]. Los geles de carbono son considerados como el material más promisorio para esta aplicación debido a su red estructural tridimensional $(5-10 \mathrm{~nm})$, alta 
área superficial específica $\left(400-2000 \mathrm{~m}^{2} / \mathrm{g}\right)$, baja densidad $\left(0,3-1 \mathrm{~g} / \mathrm{cm}^{3}\right)$, buena conductividad eléctrica (5-50S/cm), alta pureza (> 99,5\% carbón) y un tamaño de poros adaptable a electrolitos convencionales lo que se manifiesta en una mayor densidad de potencia [14].

Los geles de carbono son materiales sintéticos obtenidos a partir de compuestos fenólicos en solución acuosa. Los precursores más utilizados para la preparación de geles orgánicos son resorcinol-formaldehído [15], [16] melaminaformaldehído [17], furfural-fenol [18-20], fenolformaldehído [21], etc., utilizando diferentes disolventes (agua, metanol, etanol y otros disolventes orgánicos) y utilizando catalizadores básicos (carbonatos e hidróxidos) o ácidos (orgánicos e inorgánicos). El tipo de catalizador inicia la reacción de adición con la formación de los aniones. El tipo de catalizador y tipo de disolvente utilizado definen el $\mathrm{pH}$ inicial de la disolución, este valor es una variable importante en la preparación de los geles orgánicos porque controla la cinética de polimerización, el entrecruzamiento y, por tanto, la morfología, el tamaño de partícula y la porosidad de los geles finales [22]. A bajos $\mathrm{pH}$ se promueven las reacciones de condensación, obteniéndose un alto entrecruzamiento y una estructura muy fuerte, mientras que a altos $\mathrm{pH}$ se obtiene geles con elevado volumen de poros y área superficial. A pH muy altos se dificulta la polimerizacióncondensación y se puede causar un colapso de la nanoestructura, dando lugar a geles de carbón sin un desarrollo poroso [23]. Los valores de $\mathrm{pH}$ más utilizados para la síntesis de geles de carbón varían entre 5,4 y 7,6. La estructura final de los geles de carbono se obtiene luego del secado (subcrítico, supercrítico o criogénico), y posterior carbonización y/o activación (física o química) con el fin de aumentar el área superficial, y también se pueden incorporar heteroátomos tales como oxígeno, nitrógeno y/o fósforo, que puedan promover efectos pseudocapacitivos originados por propiedades de electrón aceptor/donador [24]. La activación química es la más utilizada debido a que se alcanzan áreas superficiales específicas mayores a $1000 \mathrm{~m}^{2} / \mathrm{g}$ donde los agentes activantes más comunes son $\mathrm{KOH}$ [25-29], $\mathrm{K}_{2} \mathrm{CO}_{3}$ [30-33], $\mathrm{ZnCl}_{2}[30,34,35]$ y $\mathrm{H}_{3} \mathrm{PO}_{4}[30,36-42]$. El $\mathrm{KOH}$ como agente activante tiende a producir una estructura más microporosa con poros anchos, el $\mathrm{K}_{2} \mathrm{CO}_{3}$ ha sido utilizado recientemente por ser ambientalmente amigable y seguro, el $\mathrm{ZnCl}_{2}$ es un agente que tiende un mayor desarrollo mesoporoso y el $\mathrm{H}_{3} \mathrm{PO}_{4}$ proporciona una distribución de poros más heterogénea con posibilidad de inclusión de heteroátomos de fósforo y es de fácil recuperación $[40,43]$. La activación con $\mathrm{H}_{3} \mathrm{PO}_{4}$ es una buena técnica para mejorar la densidad electrónica de los materiales carbonosos y aumentar la densidad de energía y potencia de un supercondensador [42]. Recientemente algunos investigadores [39,41,42] se han inclinado por activar diferentes precursores con $\mathrm{H}_{3} \mathrm{PO}_{4}$ debido a la posibilidad de obtener una estructura micro/mesoporosa y con una presencia de heteroátomos de fósforo para su aplicación como electrodos de supercondensadores. En estos trabajos los materiales se activaron a una temperatura entre 400 y $600^{\circ} \mathrm{C}$, los valores máximos de capacidad específica variaron entre 125 y $175 \mathrm{~F} / \mathrm{g}$ en medio acuoso. Los materiales preparados presentaron una buena estabilidad después de miles de ciclos (1000-5000) y una buena retención de la capacidad al aumentar la densidad de corriente hasta valores entre 2 y $3,5 \mathrm{~A} / \mathrm{g}$. En estas investigaciones el buen desempeño electroquímico es atribuido a la sinergia presentada entre las características superficiales y los heteroátomos de $\mathrm{N}, \mathrm{O}$ y $\mathrm{P}$ presentes en la estructura carbonosa que mejoran la humectabilidad del electrodo. La estructura electrónica de los grupos funcionales N/O/P y la conjugación de los electrones- $p$ proporcionan sitios de reacción farádica reversible $[42,44]$. Las especies $P$ también mejoran la densidad de energía de los supercondensadores debido a la obstrucción de los sitios activos de la oxidación electroquímica por parte de los grupos de fósforo.

La lignina es el segundo biopolímero más abundante después de la celulosa, es de bajo costo y de naturaleza fenólica. También se ha reconocido como un excelente precursor del carbón activado y bio-absorbente por su alto peso molecular y estructura polimérica con presencia de grupos funcionales carboxílicos, carbonilos e hidroxilos [45]. El mecanismo de reacción de la lignina con el formaldehido es similar al que ocurre entre el fenol y el formaldehido, son reacciones de condensación donde el fenol reacciona primero, catalizado por un medio alcalino para formar fenol metiolato. El fenol alberga sitios libres de reactividad en posiciones orto y para a diferencia de la lignina que se basa en anillos aromáticos altamente sustituidos lo que se traduce en menos sitios activos y un mayor impedimento estérico. Los grupos hidroxilos de la lignina están bloqueados por grupos funcionales quedando solo disponibles las posiciones meta 
para la reacción con el formaldehido en los anillos aromáticos de la lignina, como esquematiza Grishechko [47] en su trabajo. Esta característica de la lignina es la razón por la cual no se pueda usar sola y debe ser mezclada con otro precursor para la preparación de geles.

En este trabajo, se prepararon xerogeles activados de carbono obtenidos a partir de la sustitución parcial de resorcinol por lignina y el efecto de la relación de compuesto fenólico/catalizador, con el objetivo de evaluar su desempeño electroquímico como electrodos para supercondensadores. Los materiales de carbono a base de lignina con porosidad controlada al ser implementados en aplicaciones relacionadas con la energía, pueden llegar a mejorar la sostenibilidad ambiental mediante la reducción de la huella de carbono e impulsando la economía de las fábricas productoras de lignina [46].

\section{Metodología Experimental}

Preparación de xerogeles de carbono activados La lignina fue suministrada por Propal (Cali, Colombia) obtenida por extracción de licor negro del proceso de pulpeo alcalino, fue obtenida de acuerdo a la metodología propuesta por Castro [48]. Los xerogeles de carbono fueron preparados por el método sol-gel, mediante la reacción de polimerización del resorcinol $(R)$ y lignina $(L)$ con formaldehido $(F)$ en agua $(W)$ usando hidróxido de sodio como catalizador (C) para facilitar la disolución de la lignina. El resorcinol fue reemplazado con lignina en un $27 \% \mathrm{~m}$, las relaciones másicas $(R+L) / F$ y $(R+L) / W$ se mantuvieron constantes en 1,86 y 0,62 , respectivamente, y se seleccionaron dos relaciones de catalizador $(R+L) / C, 90,3$ y 72,1 . Las disoluciones se llevaron a unos tubos de vidrio, los cuales se sellaron para su posterior curado a $85^{\circ} \mathrm{C}$ por 5 días y se cortaron en monolitos de aproximadamente de $1 \mathrm{~cm}$ de longitud y $0,5 \mathrm{~cm}$ de diámetro. Los monolitos se lavaron sucesivamente con etanol y se secaron a presión atmosférica y $40^{\circ} \mathrm{C}$ para así obtener xerogeles de orgánicos.

En la Tabla 1 se presentan las relaciones másicas y el $\mathrm{pH}$ de las disoluciones iniciales preparadas. Los xerogeles orgánicos fueron molidos a un tamaño entre 0,5 y $1,18 \mathrm{~mm}$, se impregnaron por una hora con ácido fosfórico a una relación másica acido/xerogel de 0,85/1 y activados por 2 horas a $450^{\circ} \mathrm{C}$. Los xerogeles activados se lavaron varias veces con agua hasta alcanzar un $\mathrm{pH}$ contante y luego se secaron a $120^{\circ} \mathrm{C}$. La serie $\mathrm{G}$ corresponde a las muestras preparadas con una relación másica de (Resorcinol + Lignina)/Catalizador de 90,3 y la serie $\mathrm{D}$ de 72,1. Los números 0 y 27 corresponden al contenido en masa de lignina incluido en la formulación de los geles.

Tabla 1. Relación de masas y pH de las disoluciones iniciales.

\begin{tabular}{ccccc}
\hline Muestra & $\begin{array}{c}\mathrm{L} /(\mathrm{R}+\mathrm{L}) * 100 \\
(\% \mathrm{~m})\end{array}$ & $\begin{array}{c}\mathrm{R} / \mathrm{L} \\
(\% \mathrm{~m})\end{array}$ & $\begin{array}{c}\mathrm{RL} / \mathrm{C} \\
(\% \mathrm{~m})\end{array}$ & $\mathbf{p H}$ \\
\hline G0 & 0,00 & - & 90,3 & 7,27 \\
D0 & 0,00 & - & 72,1 & 7,11 \\
G27 & 27,16 & 2,68 & 90,2 & 5,99 \\
D27 & 27,16 & 2,68 & 72,1 & 6,65 \\
\hline
\end{tabular}

\section{Caracterización fisicoquímica}

La densidad real de los xerogeles orgánicos se determinó por medio de un picnómetro de Helio AccuPyc II 1340 (Micromeritics) y la densidad aparente se determinó a partir de la medición del peso y el volumen de los monolitos de los xerogeles orgánicos secos. Todos los materiales fueron caracterizados por fisisorción de $\mathrm{N}_{2}$ a $-196^{\circ} \mathrm{C}$ y $\mathrm{CO}_{2} 0^{\circ} \mathrm{C}$. Las isotermas de adsorción/ desorción fueron medidas en un Autosorb 1 de Quantachrome, donde se desgasificaron a $110^{\circ} \mathrm{C}$ a vacío $\left(10^{-6} \mathrm{mbar}\right)$ por $12 \mathrm{~h}$. El área superficial específica $\left(\mathrm{S}_{\mathrm{BET}}\right)$ se determinó utilizando el modelo de Brunauer-Emmett-Teller y la distribución de tamaño de poros (PSD) se determinó utilizando el modelo teórico funcional de la densidad (DFT) a partir de los datos de la isoterma de adsorción de $\mathrm{N}_{2}$ a $-196^{\circ} \mathrm{C}$. La ecuación Dubinin-Radushkevich (DR) se aplicó a las isotermas de adsorción de $\mathrm{N}_{2}$ y $\mathrm{CO}_{2}$ para obtener los valores de volumen de los microporos $\mathrm{W}_{0}$ y tamaño de poro $\mathrm{L}_{0}$. El ancho de los poros es función de la energía de adsorción $E_{0}$. Donde $L_{0}=24 / E_{0}$, para valores de $E_{0}$ menores que $20 \mathrm{~kJ} / \mathrm{mol}$ que corresponde a anchos de poro superior a 1,3nm [49]. El volumen de nitrógeno adsorbido a presión relativas de 0,95 $\left(\mathrm{V}_{0.95}\right)$, fue considerado como el volumen total del poro apreciable obtenido de la isoterma de adsorción del $\mathrm{N}_{2}$. El volumen de mesoporo $\left(\mathrm{V}_{\text {meso }}\right)$, fue obtenido por la diferencia entre $\mathrm{V}_{0,95} \mathrm{y} \mathrm{W}_{0}\left(\mathrm{~N}_{2}\right)$ [50]. La morfología de los xerogeles de carbón activados se determinó por microscopía electrónica de barrido (SEM) usando un microscopio JEOL JSM 5910 LV operado a $10 \mathrm{kV}$ equipado con dispersión inversa y detectores de electrones secundarios, junto con 
espectrometría de energía dispersiva (EDS). La química superficial de los materiales se determinó mediante la realización de espectroscopía fotoelectrónica de rayos X (XPS). Los XPS se realizaron en el equipo Thermo Scientific Escalab 250Xi: manteniendo una presión base de análisis de $10^{-10} \mathrm{mbar}$; la excitación de los fotoelectrones analizados se realizó con una fuente de rayos $X$ monocromática de $\mathrm{Al} \mathrm{Ka}(1486,68 \mathrm{eV})$, radio de análisis de $650 \mu \mathrm{m}$; al igual que las condiciones de análisis para las zonas de alta resolución (energía de paso de $20 \mathrm{eV}, 45^{\circ}$ de "take-off angle" c/paso de $0,1 \mathrm{eV})$.

\section{Caracterización electroquímica}

Las medidas electroquímicas se llevaron a cabo en un potenciostato multicanal Autolab M204 a temperatura ambiente. Se usó una configuración típica de dos y tres electrodos con $\mathrm{H}_{2} \mathrm{SO}_{4} 1 \mathrm{M}$ como electrolito. Los electrodos de trabajo se construyeron con un mezcla homogénea de xerogel de carbono activado, negro de acetileno y aglutinante (PTFE) en una relación másica $80: 10: 10$, respectivamente, sobre un papel de grafito rectangular $(2 \mathrm{~cm} \times 5 \mathrm{~cm})$ para la configuración de tres electrodos y un disco de $0,79 \mathrm{~cm}^{2}$ para la configuración de dos electrodos. La masa activa del electrodo de trabajo para la configuración de tres y dos electros fue de $26,6 \mathrm{mg}$ y $12 \mathrm{mg}$, respectivamente.

Las curvas de voltamperometría cíclica (CV) se realizaron en una configuración de tres electrodos, utilizando $\mathrm{Ag} / \mathrm{AgCl}(3 \mathrm{MKCl})$ como electrodo de referencia y platino como electrodo contador para identificar la descomposición del electrolito y efectos pseudocapacitivos [14]. Las evaluaciones de ventana se realizaron a $0,5 \mathrm{mV} / \mathrm{s}$ entre 0 $0,6,0,65,0,7,0,75,0,8$ y $0,85 \mathrm{~V}$ para evaluar la descomposición del electrolito y selección de potencial de ventana apropiado. En el rango de ventana de potencial seleccionado las curvas $\mathrm{CV}$ se realizaron a 0,5 y $10 \mathrm{mV} / \mathrm{s}$ y se determinó la capacidad gravimétrica específica, $\mathrm{C}_{\mathrm{CV}}(\mathrm{F} / \mathrm{g})$, de acuerdo a la Ecuación 1.

$$
\mathrm{C}_{\mathrm{CV}}=\frac{(\Sigma \mathrm{I} * \Delta \mathrm{t})}{\left(2 * \mathrm{~m}^{*} \Delta \mathrm{V}\right)}
$$

Donde $\Sigma I^{*} \Delta t$ es el área bajo la curva de la corriente (A) respecto al tiempo (s), m la masa del material activo (g) y $\Delta \mathrm{V}$ la ventana de potencial (V).

Las curvas de cronopotenciometría (CP) se realizaron en una configuración de dos electrodos simétricos entre 0 y $0,75 \mathrm{~V}$, rango definido a partir de las curvas $\mathrm{CV}$, y a diferentes valores de densidad de corriente, entre $0,125 \mathrm{~A} / \mathrm{g}$ y $1 \mathrm{~A} / \mathrm{g}$ equivalente a 1,2 y $9,6 \mathrm{~mA}$, respectivamente. La capacidad gravimétrica a partir de las curvas $\mathrm{CP}, \mathrm{C}_{\mathrm{CP}}(\mathrm{F} / \mathrm{g})$, se calculó de acuerdo a la Ecuación 2.

$$
\mathrm{C}_{\mathrm{CP}}=\frac{\left(\mathrm{I}_{\mathrm{d}} * \Delta \mathrm{t}\right)}{(\mathrm{m} * \Delta \mathrm{V})}
$$

Donde $I_{d}$ es la corriente de descarga $(\mathrm{A}), \Delta \mathrm{t}$ tiempo de descarga tiempo (s), m la masa del electrodo (g), y $\Delta \mathrm{V}$ el intervalo de potencial $(\mathrm{V})$.

Las curvas de espectroscopía de impedancia electroquímica (EIS) se realizaron variando la frecuencia de los iones a través de la red porosa entre $1 \mathrm{mHz}$ y $100 \mathrm{kHz}$ a una amplitud $\pm 10 \mathrm{~mA}$. La capacidad gravimétrica obtenida por EIS, se determinó por medio de la Ecuación 3 y la Ecuación 4.

$$
\begin{aligned}
& C^{\prime}=\frac{-Z^{\prime \prime}}{\left(\omega|Z|^{2}\right)} \\
& C^{\prime \prime}=\frac{Z^{\prime}}{\left(\omega|Z|^{2}\right)}
\end{aligned}
$$

Donde C' (F/g) es la parte real de la capacidad y representa la variación de la energía almacenada en función de la frecuencia, Z" es la parte compleja de la impedancia, $|Z|$ la magnitud de la impedancia y w el frecuencia angular. La pérdida de la capacidad que ocurre durante el almacenamiento de energía C" (capacidad imaginaria). A partir de las curvas de EIS también se determinó el tiempo de relajación $(t)$ a la frecuencia correspondiente a la máxima capacidad imaginaria (C") mediante la Ecuación 5 [8].

$$
\tau=\frac{1}{\left(2 * \pi^{*} \mathrm{f}\right)}
$$

\section{Resultados y Discusión}

En la Tabla 1 se puede observar que la cantidad de catalizador influye en el $\mathrm{pH}$ de la disolución y en la densidad de los monolitos obtenidos. Las disoluciones sin lignina presentaron un $\mathrm{pH}$ alrededor de 7,1, valor que disminuyó al incorporar la lignina en la disolución. El pH de la disolución D27 fue ligeramente mayor que el de la G27 lo que correspondealacantidad proporcional decatalizador adicionado y que favorece la incorporación de la lignina. Al incrementar el contenido de catalizador, se favorece el entrecruzamiento de la lignina en 
la red polimérica ya que ésta tiene una menor reactividad que el resorcinol [47]. En la Tabla 2 se presentan los valores de densidad real $\left(\rho_{\mathrm{He}}\right)$, densidad aparente $\left(\rho_{\mathrm{b}}\right)$ y porcentaje de porosidad (P). La $\rho_{\mathrm{He}}$ presentó un valor similar para todas las muestras. Sin embargo, los xerogeles orgánicos con lignina presentaron una estructura con un mayor porcentaje de porosidad respecto a las muestras sin lignina.

Tabla 2. Valores de densidad real y aparente y porcentaje de porosidad de los xerogeles orgánicos secos.

\begin{tabular}{cccc}
\hline Muestra & $\begin{array}{c}\rho_{\text {He }} \\
\left(\mathbf{g} / \mathbf{c m}^{3}\right)\end{array}$ & $\begin{array}{c}\rho_{\mathbf{b}} \\
\left(\mathbf{g} / \mathbf{c m}^{3}\right)\end{array}$ & $\begin{array}{c}\mathbf{P} \\
\mathbf{( \% )}\end{array}$ \\
G0 & 1,3366 & 0,967 & 27,65 \\
D0 & 1,0464 & 0,899 & 14,09 \\
G27 & 1,3625 & 0,552 & 59,49 \\
D27 & 1,3731 & 0,525 & 61,77 \\
\hline
\end{tabular}

En la Figura 1a se representan las isotermas de adsorción/desorción de $\mathrm{N}_{2}$ a $-196^{\circ} \mathrm{C}$ de los xerogeles de carbono activados (GA0, DA0, GA27 y DA27). Los xerogeles con lignina presentaron una presencia de mesoporos debido al ciclo de histéresis formado a altas presiones relativas. Las muestras GA0 y DA0 se pueden clasificar como tipo I según la IUPAC, típica de sólidos microporosos, y las muestras GA27 y DA27 como tipo I-IV, correspondiente a materiales micro-mesoporosos. En la Figura $1 \mathrm{~b}$ se representan las isotermas de adsorción de $\mathrm{CO}_{2}$ a $0^{\circ} \mathrm{C}$, en las cual no se observa un efecto de la relación de catalizador sobre los microporos estrechos o ultramicroporos (menos de 0,7 - 0,8nm de ancho). Las distribuciones de tamaño de poro (PSD) son de distribución similares para los xerogeles de carbón activados de ambas series.

En la Tabla 3 se presentan los valores de área superficial específica $\left(\mathrm{S}_{\mathrm{BET}}\right)$, tamaño $\left(\mathrm{L}_{0}\right)$ y volumen de microporos $\left(\mathrm{W}_{0}\right)$, volumen total de poros $\left(\mathrm{V}_{0.95}\right)$, volumen de mesoporos $\left(\mathrm{V}_{\text {meso }}\right)$ obtenidos a partir de las isotermas de adsorción de $\mathrm{CO}_{2}$ a $0^{\circ} \mathrm{C}$ y $\mathrm{N}_{2}$ a $-196^{\circ} \mathrm{C}$. El valor de $\mathrm{W}_{0}\left(\mathrm{~N}_{2}\right)>\mathrm{W}_{0}\left(\mathrm{CO}_{2}\right)$ para todas las muestras, lo que sugiere un alto grado de activación y una distribución de tamaño heterogéneo de los microporos. En este caso el $\mathrm{N}_{2}$ es capaz de llenar el volumen total de microporos, obtenido de la adsorción a $-196^{\circ} \mathrm{C}$, mientras que el
$\mathrm{CO}_{2}$ a $0^{\circ} \mathrm{C}$ sólo llena los microporos estrechos [51]. La muestra sin lignina y con un menor contenido de catalizador (GA0) presentó un mayor desarrollo microporoso, respecto a la muestra DA0, y por consiguiente un mayor valor de $\mathrm{S}_{\mathrm{BET}}$. Las muestras con lignina presentaron una mayor microporosidad, obteniendo un valor máximo de $\mathrm{S}_{\mathrm{BET}}$ de 1288 correspondiente a la muestra GA27. La muestra DA27 presentó el mayor volumen total de poros debido al aporte del volumen de los mesoporos. Las muestras preparadas con lignina presentaron valores de $S_{B E T}$ y microporosidad ligeramente más altos que los presentados por Hayashi y col. para carbones activados a partir de la lignina por activación química con $\mathrm{H}_{3} \mathrm{PO}_{4}$ a $600^{\circ} \mathrm{C}$ y una relación de impregnación de $1 / 1\left(1000 \mathrm{~m}^{2} / \mathrm{g}\right)$ [52] y los obtenidos por Saha y col. activados con $\mathrm{KOH}$ a $200 \% \mathrm{~m}$ y $1000^{\circ} \mathrm{C}\left(1148 \mathrm{~m}^{2} / \mathrm{g}\right)$ [46]. En la literatura se reportan relaciones de volumen de microporos y volumen de mesoporos $\left(\mathrm{W}_{0}\left(\mathrm{~N}_{2}\right) / \mathrm{V}_{\text {meso }}\right)$ entre $1 \mathrm{y}$ 30 , la muestra DA27 presentó la relación más baja (3.1), lo cual sugiere una proporción ligeramente mayor del volumen de microporos respecto al volumen de mesoporos.

En la Figura 2 se presentan las microfotografías obtenidas para la serie $G$ y $D$. Las muestras GA0 y DA0 presentaron una estructura carbonosa continua, compacta y cerrada. Las muestras con lignina presentaron morfologías con grandes huecos o macroporos debido a la alta interacción entre el agente químico y lignina, la cual tiene una morfología superficial tri-dimensional como se muestran Castro y Quintana en [48]. La formación de macroporos en xerogeles es inducida por el gran tamaño de la molécula de lignina, que no permite un buen empacado y también evita el encogimiento $[48,53,54]$. La lignina tiene sitios menos reactivos por masa que las dadas entre el resorcinol y formaldehído por reacciones de adición y de condensación a través de un número de enlaces éter $(\mathrm{C}-\mathrm{O}-\mathrm{C})$ y enlaces carbono-carbono (C-C) que están distribuidos al azar $[47,55,56]$. La presencia de macroporos es más evidente en la muestra GA27 que en la DA27, ya que un mayor contenido de catalizador en la serie D que promueve la formación de una estructura más estrecha y menor accesibilidad al interior del xerogel orgánico, lo cual influye levemente en la disminución de la microporosidad. 

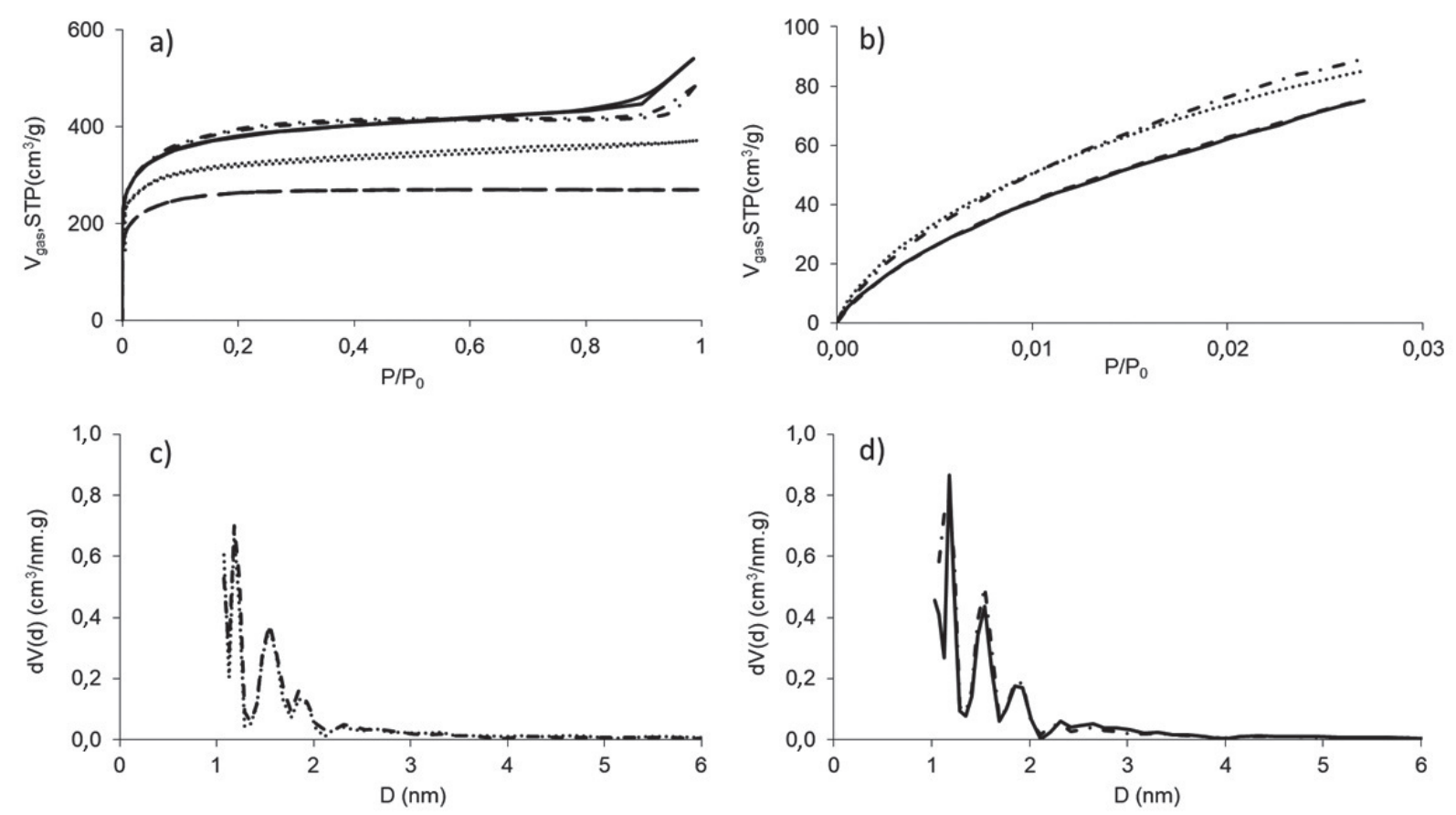

Figura 1. a) Isotermas de adsorción-desorción de $\mathrm{N}_{2}$ a $-196^{\circ} \mathrm{C}$, b) Isotermas de adsorción de $\mathrm{CO}_{2}$ a $0^{\circ} \mathrm{C}, \mathrm{c}$ ) y d)

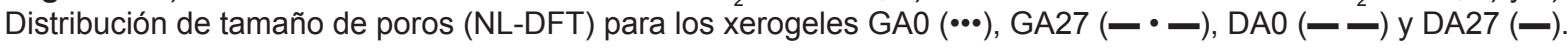

Tabla 3. Propiedades texturales para los xerogeles de carbono activados.

\begin{tabular}{|c|c|c|c|c|c|c|c|c|}
\hline Muestra & $\begin{array}{c}S_{B E T}^{1} \\
\left(m^{2} / g\right)\end{array}$ & $\begin{array}{l}W_{0}\left(N_{2}\right)^{2} \\
\left(\mathrm{~cm}^{3} / g\right)\end{array}$ & $\begin{array}{c}\mathrm{W}_{0}\left(\mathrm{CO}_{2}\right)^{2} \\
\left(\mathrm{~cm}^{3} / \mathrm{g}\right)\end{array}$ & $\begin{array}{c}V_{0,95}{ }^{3} \\
\left(\mathrm{~cm}^{3} / \mathrm{g}\right)\end{array}$ & $\begin{array}{c}V_{\text {meso }}{ }^{4} \\
\left(\mathrm{~cm}^{3} / \mathrm{g}\right)\end{array}$ & $\begin{array}{c}\mathbf{W}_{0}\left(\mathbf{N}_{2}\right) l \\
\mathbf{V}_{\text {meso }}\end{array}$ & $\begin{array}{c}\mathrm{L}_{0}\left(\mathrm{~N}_{2}\right)^{5} \\
\left(\mathrm{~nm}^{2}\right)\end{array}$ & $\begin{array}{c}\mathrm{L}_{0}\left(\mathrm{CO}_{2}\right)^{5} \\
(\mathrm{~nm})\end{array}$ \\
\hline GA0 & 1101 & 0,455 & 0,290 & 0,519 & 0,065 & 7,0 & 1,43 & 0,63 \\
\hline DA0 & 893 & 0,362 & 0,258 & 0,382 & 0,020 & 18,4 & 1,32 & 0,68 \\
\hline GA27 & 1288 & 0,584 & 0,310 & 0,613 & 0,029 & 20,3 & 1,41 & 0,66 \\
\hline DA27 & 1272 & 0,578 & 0,244 & 0,765 & 0,188 & 3,1 & 1,34 & 0,64 \\
\hline
\end{tabular}

${ }^{1}$ Área superficial BET, ${ }^{2}$ Volumen de microporos, ${ }^{3}$ Volumen de nitrógeno adsorbido a presión relativas de 0,95 , ${ }^{4}$ Volumen de mesoporos y ${ }^{5}$ Tamaño de microporos.

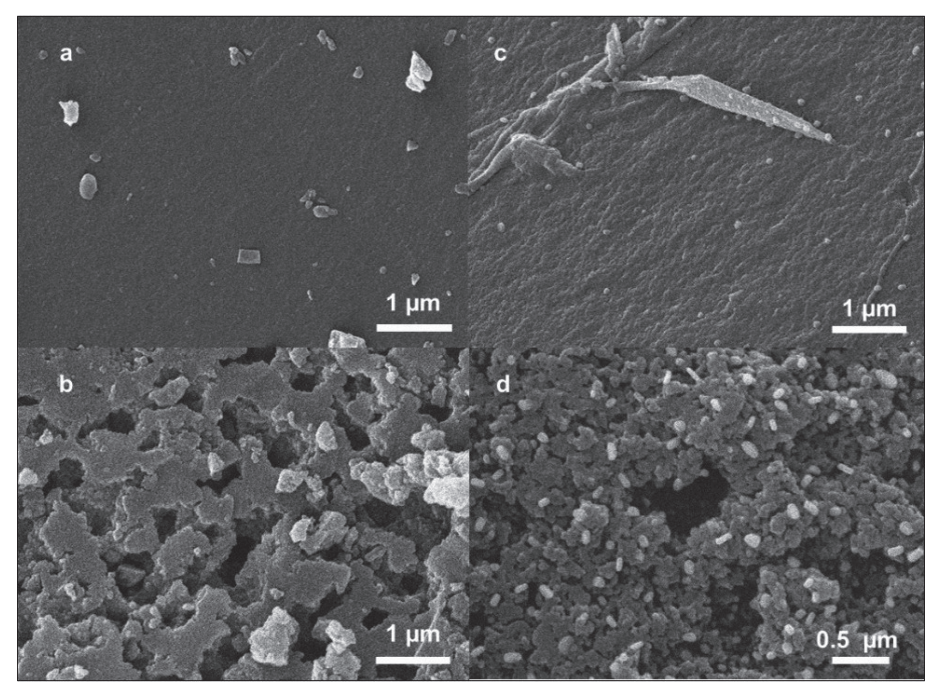

Figura 2. Micrografías SEM a 20kV y X20000 para a) GA0 y b) GA27 y a 20 kV y X30000 para c) DA0 y d) DA27. 
La química superficial de los materiales se determinó mediante la realización de espectroscopía fotoelectrónica de rayos X (XPS). En el caso de los materiales carbonosos preparados en este trabajo las regiones de interés a alta resolución fueron $\mathrm{C}_{1 \mathrm{~s}}$, $\mathrm{O}_{1 \mathrm{~s}}, \mathrm{~N}_{1 \mathrm{~s}}$ y $\mathrm{P}_{2 \mathrm{p}}$. La energía de enlace del pico de la región $\mathrm{C}_{1 \mathrm{~s}}$ a $284,6 \mathrm{eV}$ corresponde a el enlace $\mathrm{C}=\mathrm{C}$ (aromático-alifático) y se usó como pico de referencia para conocer la posición de los demás picos del espectro. Los espectros obtenidos, una vez restada la línea base, se deconvolucionaron por medio de un método iterativo de ajuste, usando funciones de suma asimétricas Gaussianas-Lorentzianas para determinar el número de componentes, picos de energía de enlace y el área de los mismos. El espectro $C_{1 \mathrm{~s}}$ está representado por sietes energías de enlaces: P-C ( 283,7eV) [57], $\mathrm{C}=\mathrm{C}(\sim 284,6 \mathrm{eV})$, C-C $\mathrm{sp}^{3}$ alifático $(\sim 285,3 \mathrm{eV}), \mathrm{R}-\mathrm{OH}$ y C-O-C y/o $\mathrm{C}-\mathrm{O}-\mathrm{P}(\sim 286,3 \mathrm{eV}), \mathrm{C}=\mathrm{O}+>\mathrm{C}=\mathrm{O}(\sim 287,5 \mathrm{eV}), \mathrm{COOH}$ + -C(O)-O-C ( 289,3eV) y p-p* ( 291,6eV) [58]. El espectro $\mathrm{O}_{1 \mathrm{~s}}$ está representado por cinco energías de enlace: $\mathrm{O}=\mathrm{C}+\mathrm{O}=\mathrm{P}(\sim 530,5 \mathrm{eV})$, tipo quinona $\mathrm{C}=\mathrm{O}$ $(\sim 531,3 \mathrm{eV}), \mathrm{O}-\mathrm{C}+\mathrm{COH}+\mathrm{C}-\mathrm{O}-\mathrm{C}+\mathrm{P}-\mathrm{O}(\sim 532,4 \mathrm{eV})$, $\mathrm{C}-\mathrm{OH}$ y/o P-O-P $(\sim 533,3 \mathrm{eV})$, y oxígeno quimisorbido y $\mathrm{H}_{2} \mathrm{O}(\sim 534,5 \mathrm{eV})$ [58], [59]. El espectro $\mathrm{P}_{2 p}$ está representado por tres energías de enlace: $\mathrm{C}_{3}-\mathrm{PO}$ $(\sim 132,2 \mathrm{eV}), \mathrm{C}-\mathrm{PO}_{3} \circ \mathrm{C}_{2}-\mathrm{PO}_{2}(\sim 133,5 \mathrm{eV})$ y grupos tipo C-O-P tales como (CO) ${ }_{3} \mathrm{PO},(\mathrm{CO})_{2} \mathrm{PO}_{2}$ y $(\mathrm{CO}) \mathrm{PO}_{3}$ $(\sim 134,5 \mathrm{eV})$ [59], donde el pico de mayor presencia corresponde a $\sim 133,5 \mathrm{eV}$. El espectro $\mathrm{N}_{1 \mathrm{~s}}$ solamente se evidenció en la muestra GA27 y está representado por tres energías de enlace: $\mathrm{N}-6$ y $\mathrm{N}=\mathrm{P}(\sim 398,1 \mathrm{eV})$, $\mathrm{N}-5$ ( 399. eV) y N-Q ( 401.9 eV) [60]. En la Tabla 4 se presentan los resultados de la deconvolución de los espectros para las regiones $C_{1 s}, O_{1 s}, N_{1 s}$ y $P_{2 p}$, y la composición elemental superficial de las muestras preparadas. Las muestras sin lignina presentaron similares porcentajes de $\mathrm{O}_{1 \mathrm{~s}}$ y $\mathrm{P}_{2 \mathrm{p}}$ superficial, $\sim 19 \% \mathrm{~m}$ y $\sim 4,3 \% \mathrm{~m}$, respectivamente. La incorporación de la lignina promovió una disminución del porcentaje de fósforo en la superficie $(\sim 3,3 \% \mathrm{~m})$, lo cual puede ser asociado a una mayor reactividad de la lignina con el ácido fosfórico durante el proceso de activación. El contenido de $\mathrm{N}_{1 \mathrm{~s}}$ en la muestra GA27 fue relativamente bajo $0,5 \% \mathrm{~m}$, y corresponde principalmente a la presencia de grupos funcionales $\mathrm{N}-5$.

En la Figura 3 se presentan las curvas de voltamperometría cíclica a $0,5 \mathrm{mV} / \mathrm{s}_{2} \quad \mathrm{H}_{2} \mathrm{SO}_{4} \quad 1 \mathrm{M}$ realizadas utilizando una configuración tres electrodos para las muestras GA27 y DA27 a diferentes ventanas de potencial.
Tabla 4. Resultados de deconvolución del espectro XPS para $\mathrm{C}_{1 \mathrm{~s}}, \mathrm{O}_{1 \mathrm{~s}}, \mathrm{~N}_{1 \mathrm{~s}}$ y $\mathrm{P}_{2 \mathrm{p}}$ y composición elemental superficial de xerogeles de carbono activados.

\begin{tabular}{|c|c|c|c|c|c|}
\hline \multirow{2}{*}{$\begin{array}{l}\text { Región } \\
\text { Elemento }\end{array}$} & \multicolumn{5}{|c|}{$\%$ pico } \\
\hline & Posición (eV) & GA0 & DAO & GA27 & DA27 \\
\hline \multirow{8}{*}{$C_{1 s}$} & 283,9 & 8 & 6,7 & 5,7 & 11,2 \\
\hline & 284,6 & 45,1 & 48,9 & 45,6 & 41,8 \\
\hline & 285,2 & 18,1 & 14,6 & 18,9 & 18,9 \\
\hline & 286,4 & 14,4 & 11,1 & 13,2 & 11,5 \\
\hline & 288 & 6,2 & 7 & 8,5 & 5,9 \\
\hline & 289,5 & 4,6 & 6,1 & 4,8 & 5,2 \\
\hline & 291,3 & 3,5 & 5,6 & 3,4 & 5,5 \\
\hline & $\% \mathrm{~m} / \mathrm{m}$ & 76,7 & 77,3 & 80,5 & 78,9 \\
\hline \multirow{6}{*}{$\mathrm{O}_{1 \mathrm{~s}}$} & 530,5 & 11,2 & 13,7 & 8 & 10,2 \\
\hline & 531,3 & 19 & 21,3 & 15,9 & 17 \\
\hline & 532,4 & 25,9 & 26,7 & 22,8 & 21,8 \\
\hline & 533,4 & 33,2 & 26,2 & 40,6 & 40,1 \\
\hline & 534,6 & 10,7 & 12,1 & 12,6 & 10,8 \\
\hline & $\% \mathrm{~m} / \mathrm{m}$ & 19,2 & 18,3 & 15,8 & 17,4 \\
\hline \multirow{4}{*}{${ }_{N}^{1 s}$} & 396,9 & - & - & 13,2 & - \\
\hline & 401,9 & - & - & 17,7 & - \\
\hline & 399,2 & - & - & 69,1 & - \\
\hline & $\% \mathrm{~m} / \mathrm{m}$ & - & - & 0,5 & - \\
\hline \multirow{4}{*}{$P_{2 p}$} & 132,2 & 12,6 & 13,4 & 10,4 & 11,7 \\
\hline & 133,4 & 57 & 52,7 & 48,8 & 60,5 \\
\hline & 134,5 & 30,5 & 33,9 & 40,8 & 27,7 \\
\hline & $\% \mathrm{~m} / \mathrm{m}$ & 4,1 & 4,4 & 3,2 & 3,6 \\
\hline
\end{tabular}

La evaluación de ventana se realizó para las muestras preparadas con lignina, ya que presentaron un mejor desarrollo poroso y superficial. A una ventana de potencial entre 0 y $0,85 \mathrm{~V}$ se observó en ambas muestras una contribución farádica a altos voltajes debido a la descomposición del electrolito. Las curvas a una ventana de potencial entre $0 \mathrm{y}$ $0,75 \mathrm{~V}$ presentaron un comportamiento similar al comportamiento ideal de un condensador, forma rectangular e imagen espejo para ambas muestras [14]. Todas las curvas presentaron presencia de picos, entre 0,3 y $0,4 \mathrm{~V}$, relacionados con reacciones de óxido-reducción que promueven un aumento de la capacidad entre compuestos electroquímicamente activos [61]. En el rango de potencial entre 0 y $0,6 \mathrm{~V}$ es muy estrecho e interfiere con los efectos pseudocapacitivos. En los materiales de carbono, 
los grupos funcionales considerados como responsables de la pseudocapacidad son los de alto contenido de oxígeno de carácter básico tales como las quinonas [62]. Adicionalmente, todas las muestras presentaron heteroátomos de fósforo en su superficie, los cuales forman puentes de oxígeno (C-O-P) que favorecen la heterogeneidad de la

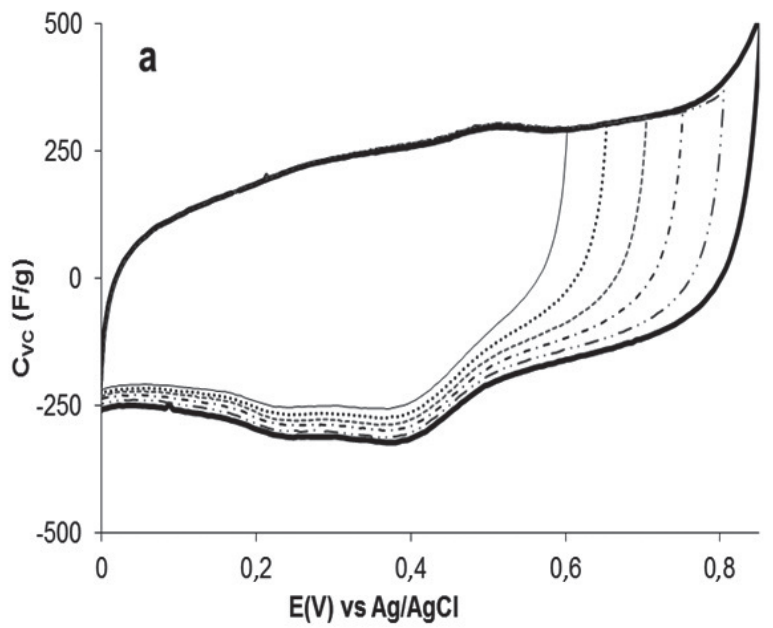

superficie carbonosa y favorece positivamente los efectos pseudocapacitivos [42]. La muestra GA27 fue la única que presentó un contenido superficial de nitrógeno, los grupos funcionales nitrogenados ayudan a la transferencia de electrones y a un aumento de la psedudocapacidad, especialmente los grupos N-Q [63].

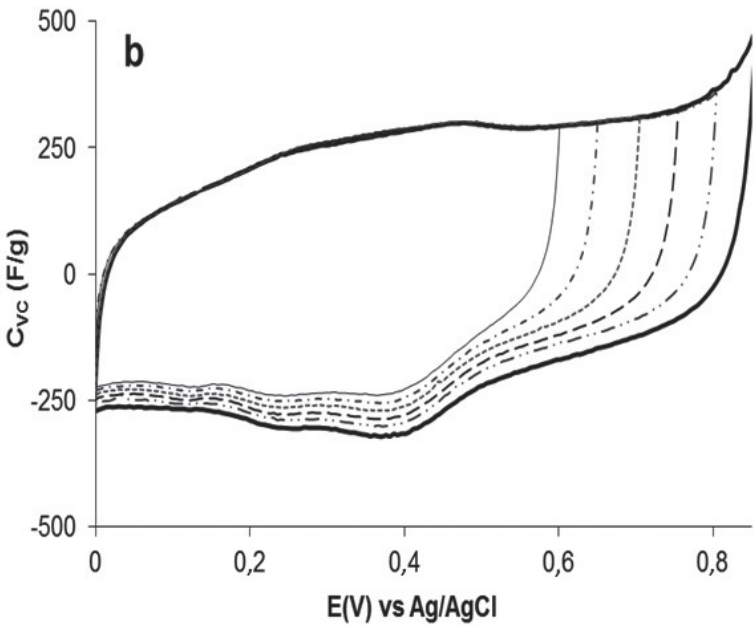

Figura 3. Curvas de voltamperometría cíclica para a) GA27 y b) DA27 en una configuración de tres electrodos en $\mathrm{H}_{2} \mathrm{SO}_{4} 1 \mathrm{M}$ a diferentes ventanas de potencial.

En la Figura $4 a$ y Figura $4 \mathrm{~b}$ se comparan las curvas de voltamperometría cíclica para ambas series (G y D) entre 0 y $0,75 \mathrm{~V}$ a 0,5 y $10 \mathrm{mV} / \mathrm{s}$, respectivamente. Las curvas $\mathrm{CV}$ a $0,5 \mathrm{mV} / \mathrm{s}$ de los xerogeles activados presentaron una forma rectangular y cercana a la forma ideal de un supercondensador, sin embargo las curvas de los xerogeles sin lignina fueron más estrechas y sin presencia de picos de oxido-reducción. Al incrementar la velocidad de barrido a $10 \mathrm{mV} / \mathrm{s}$ se observa una inclinación de las curvas que se relaciona con un alejamiento de la idealidad.

En la Figura 5a se comparan las curvas de cronopotenciometría a $0,125 \mathrm{~A} / \mathrm{g}$ entre 0 y $0,75 \mathrm{~V}$ en una configuración de dos electrodos, en la cual se observa que la muestra DA27 tiene una forma triagular simétrica, lo que indica que la carga es muy similar a la descarga y que tiene un comportamiento cercano al ideal [64]. En la Figura $5 b$ se presenta la variación de la capacidad gravimétrica a partir de las curvas CP respecto al incremento de corriente entre 1 y $10 \mathrm{~mA}$ en una configuración de dos electrodos. En esta figura se observa que al aumentar la corriente los valores de capacidad son más altos para la serie D respecto a la serie $\mathrm{G}$, debido a que posiblemente el tamaño de poro de la serie $D(1,3 \mathrm{~nm})$ se ajuste más al tamaño del ion del electrolito. A altas corrientes la muestra DA27 presentó el mejor desempeño electroquímico debido a la presencia de mesoporos que permiten el transporte de los iones. En la Figura $5 \mathrm{c}$ se presentan el diagrama de Nyquist obtenido a partir de los análisis de EIS en una celda de dos electrodos para los xerogeles de carbono activados. Los materiales carbonosos se caracterizan por tener una parte resistiva a altas frecuencias (semicírculo) y un comportamiento capacitivo a bajas frecuencias $(<1 \mathrm{~Hz})$ [65]. La muestra DA27 presentó un semicírculo más definido y amplio respecto a las otras muestras, lo cual puede estar asociado con una resistencia interna mayor debido a una estructura porosa heterogénea y estrecha [66]. En la región media se observa un efecto difusivo más amplio en la muestra GA27 respecto a la DA27, que puede estar relacionado con la presencia de mesoporos en la muestra DA27 que permite una mejor migración de los iones desde el electrolito al centro del electrodo a altas velocidades [67] carbon black (CB. En la región de baja frecuencia la penetración de los iones en los poros es casi completa y el proceso de formación de la doble capa electroquímica es lento, una línea vertical muestra el dominio del comportamiento capacitivo 
en la interfase de carbono-electrolito, lo que sugiere que la muestra DA27 tiende a ser más conductiva que la GA27, lo cual también es acorde con los valores de ángulo de fase. De acuerdo a los análisis XPS, las muestras presentaron un pico representativo en $133,5 \mathrm{eV}$, este pico proporciona una mayor contribución de átomos de oxígeno que mejoran la mojabilidad de la superficie carbonosa y el desempeño electroquímico [59], este pico

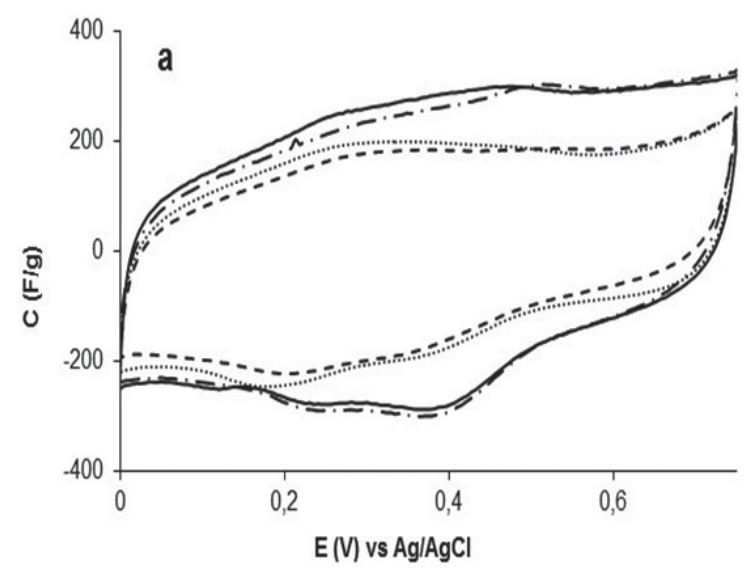

tiene una mayor representación para la muestra DA27 $(62,7 \%)$ respecto a las otras $(48,8-57 \%)$. En la Figura $5 d$ se presentan las curvas de variación de la pérdida de la capacidad (C") respecto a la frecuencia, en las cuales se observa un máximo que corresponde a la frecuencia en la que se obtiene la constante de tiempo de relajación, indicando el tiempo necesario para almacenar y entregar la energía del supercondensador.

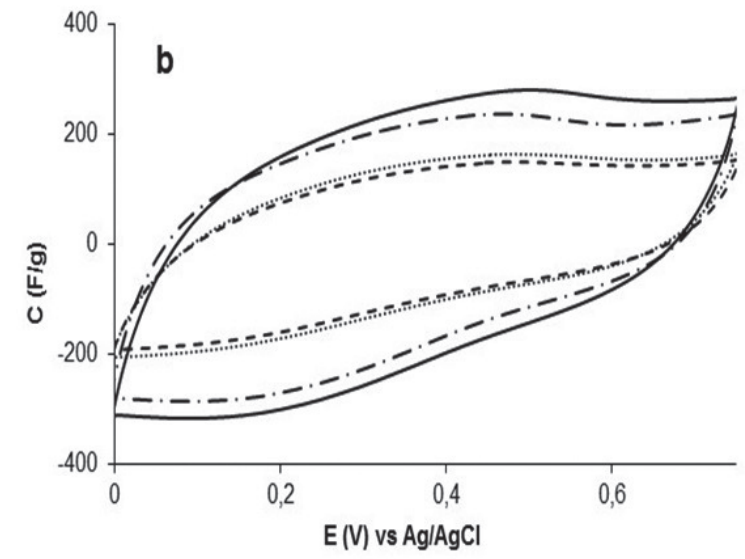

Figura 4. Curvas VC entre 0 y $0,75 \mathrm{~V}$ en $\mathrm{H}_{2} \mathrm{SO}_{4} 1 \mathrm{M}$ en una celda de tres electrodos a a) $0,5 \mathrm{mV} / \mathrm{s}$ y b) $10 \mathrm{mV} / \mathrm{s} \mathrm{para}$

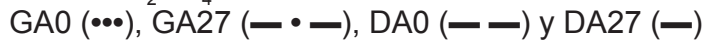
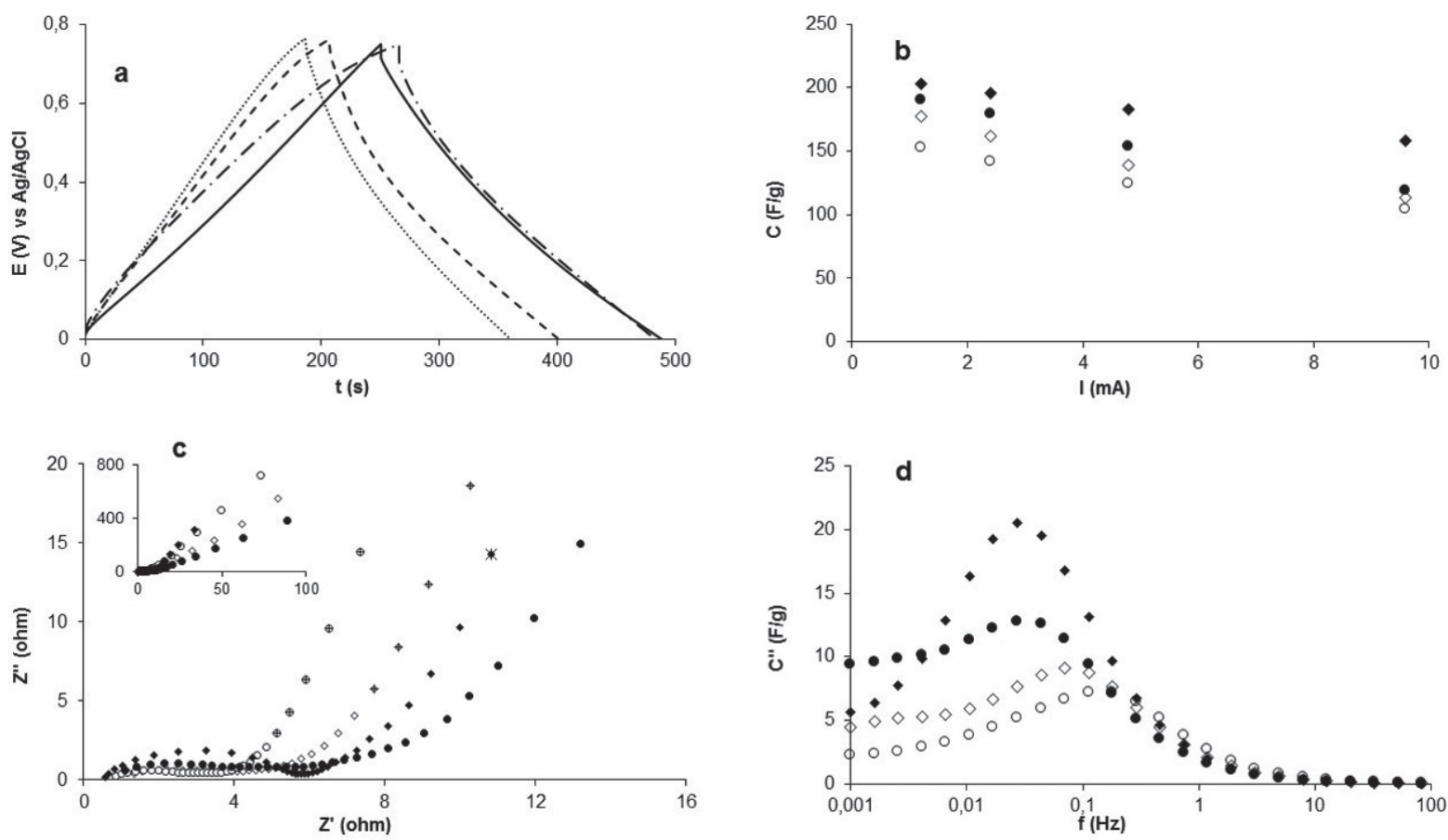

Figura 5. Comportamiento electroquímico en una celda de dos electrodos en $\mathrm{H}_{2} \mathrm{SO}_{4} 1 \mathrm{M}$ para xerogeles de carbono activados: a) Curvas CP a 0,125A/g para GA0 (•••), GA27 ( - - -), DA0 ( — - ) y DA27 ( -), b) Variación de la capacidad con la corriente $\left(\mathrm{C}_{\mathrm{CP}}\right)$, c) Diagrama de Nyquist región a alta frecuencia y rango completo (figura insertada)

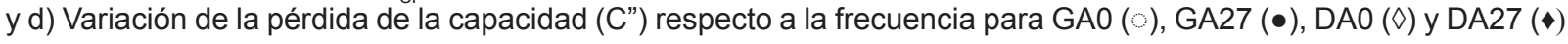


rev.ion. 2017;30(2):17-30. Bucaramanga (Colombia)

Tabla 5. Características electroquímicas de los xerogeles de carbono activados en configuraciones de dos y tres electrodos en una solución de $\mathrm{H}_{2} \mathrm{SO}_{4} 1 \mathrm{M}$

\begin{tabular}{|c|c|c|c|c|c|c|c|c|c|}
\hline \multirow{3}{*}{ Muestra } & \multirow{2}{*}{\multicolumn{2}{|c|}{$\begin{array}{c}\begin{array}{c}\text { Tres } \\
\text { electrodos }\end{array} \\
\mathrm{C}_{\mathrm{vc}}(\mathrm{F} / \mathrm{g}) \\
\end{array}$}} & \multicolumn{7}{|c|}{$\begin{array}{c}\text { Dos } \\
\text { electrodos }\end{array}$} \\
\hline & & & \multirow{2}{*}{$\begin{array}{c}R_{\mathrm{s}}(\Omega) \\
100 \mathrm{kHz}\end{array}$} & \multirow{2}{*}{$\begin{array}{c}\text { ESR } \\
(\Omega)\end{array}$} & \multirow[b]{2}{*}{ (s) } & \multirow{2}{*}{$\begin{array}{c}-\left({ }^{\circ}\right) \\
1 \\
\mathrm{mHz}\end{array}$} & \multirow{2}{*}{$\begin{array}{c}\mathrm{C}^{\prime a}(\mathrm{~F} / \mathrm{g}) \\
1 \mathrm{mHz}\end{array}$} & \multicolumn{2}{|c|}{$\mathrm{C}_{\mathrm{CP}}^{\mathrm{a}}(\mathrm{F} / \mathrm{g})$} \\
\hline & $\begin{array}{c}0,5 \\
\mathrm{mV} / \mathrm{s}\end{array}$ & $\begin{array}{c}10 \\
\mathrm{mV} / \mathrm{s}\end{array}$ & & & & & & $1,2 \mathrm{~mA}$ & $\begin{array}{l}9,6 \\
\mathrm{~mA}\end{array}$ \\
\hline GA0 & 165 & 121 & 0,84 & 5,37 & 1,4 & 84,21 & 91 & 153 & 104 \\
\hline DAO & 151 & 111 & 1,35 & 6,95 & 2,3 & 81,39 & 118 & 177 & 113 \\
\hline GA27 & 222 & 182 & 1,10 & 9,80 & 5,8 & 77,05 & 164 & 190 & 118 \\
\hline DA27 & 226 & 208 & 0,57 & 10,02 & 5,8 & 83,84 & 211 & 203 & 158 \\
\hline
\end{tabular}

En la Tabla 5 se presentan las características electroquímicas de los xerogeles de carbono activados en configuraciones de dos y tres electrodos a partir de la Figura 4 y Figura $5 a$. La muestra DA27 presentó los valores de capacidad específica más altos en las diferentes técnicas utilizadas. La retención de la capacidad a la corriente más alta respecto a la más baja fue de $62 \%$ para la muestra GA27 y $78 \%$ para la muestra DA27, esta diferencia puede estar relacionada con la baja relación entre el volumen de microporos y el volumen de mesoporos de la muestra DA27 $\left(\mathrm{W}_{0}\left(\mathrm{~N}_{2}\right) / \mathrm{N}_{\text {meso }}=3,1\right)$, que facilita el movimiento de los iones dentro de los poros. En la región de alta frecuencia de la Figura $5 \mathrm{c}$ se puede determinar el valor de la resistencia en serie equivalente (ESR equivalent serie resistance), la cual representa la suma de la resistencia de contacto, la resistencia de transferencia de carga y la resistencia la difusión en los poros del electrolito durante el proceso electroquímico. La ESR se obtiene de la extrapolación de la línea vertical a frecuencias media-baja del diagrama de Nyquist y su corte con la abscisa [68]. Los xerogeles de carbono activados con lignina presentaron una ERS mayor que sus equivalentes sin lignina, $\sim 10 \mathrm{~W}$ respecto a $\sim 6 \mathrm{~W}$, respectivamente. El tiempo de relajación $(\tau)$ indica la rapidez con la que puede cargarse o descargarse un condensador, las muestras DA27 y GA27 presentaron tiempos de relajación similares, sin embargo, la mayor cantidad de energía almacenada a la menor frecuencia fue para la muestra DA27. Las muestras GA0 y DA0 presentaron los menores tiempos de relajación debido a una baja ESR. El valor de ángulo de fase $(\varphi)$ se obtiene a partir del diagrama de bode a partir de acuerdo los resultados de la EIS a la frecuencia más baja, la muestra DA27 presentó un ángulo de fase $\left(-83,4^{\circ}\right)$ más cercano a $-90^{\circ}$ respecto que el de la GA27 $\left(-77,05^{\circ}\right)$, confirmando un comportamiento cercano a un supercondensador ideal y que corresponde a los altos valores de capacidad calculados.

\section{Conclusiones}

La capacidad de almacenamiento de energía de los xerogeles de carbono activados químicamente preparados con lignina fue alrededor de $200 \mathrm{~F} / \mathrm{g}$ a la corriente más baja y varió entre 120 y $160 \mathrm{~F} / \mathrm{g}$ para la corriente más alta en medio acuoso. Incrementar el contenido de catalizador en la preparación de los xerogeles con lignina favoreció la formación de una estructura micro-mesoporosa. La activación con ácido fosfórico facilitó la incorporación de grupos funcionales oxígeno y fósforo sobre la superficie de los xerogeles y promover efectos pseudocapacitivos. La muestra DA27 es la más apropiada para su aplicación como electrodos de supercondensadores, debido que al incrementar la corriente presentó la mejor retención de la capacidad de almacenamiento de energía electroquímica. A partir de los resultados obtenidos se puede decir que los xerogeles de carbón activados a partir de lignina tienen potencial para la conversión de un material de bajo costo, renovable y confiable en un material con un mayor valor agregado.

\section{Agradecimientos}

Este trabajo es parte del proyecto "Desarrollo de electrodos para supercondensadores a partir de xerogeles de carbono funcionalizados con lignina y triazina" y es financiado por COLCIENCIAS 658-2014 (Código: 121065842324). Giovanna Diossa agradece a la UPB por el apoyo para la realización de sus estudios de maestría mediante 
la participación como pasante del proyecto interno "Xerogeles de carbono en forma de microesferas funcionalizados con lignina" 367B-03/15-21.

\section{Referencias bibliográficas}

[1] Shukla AK, Banerjee A, Ravikumar MK, Jalajakshi A. Electrochemical capacitors: Technical challenges and prognosis for future markets. Electrochim. 2012;84:165-73.

[2] Inagaki M, Konno $\mathrm{H}$, Tanaike O. Carbon materials for electrochemical capacitors. J. Power Sources. 2010;195(24):7880- 903.

[3] Sharma P, Bhatti TS. A review on electrochemical double-layer capacitors. Energ. Convers. Manag. 2010;51(12):290112.

[4] Pandolfo A, Hollenkamp A. Carbon properties and their role in supercapacitors. J. Power Sources. 2006;157(1):11-27.

[5] Adhyapak PV, Maddanimath T, Pethkar S, Chandwadkar AJ. Application of electrochemically prepared carbon nanofibers in supercapacitors. J. Power Sources. 2002;109(1):105-10.

[6] Miller JR, Simon P. Fundamentals of electrochemical capacitor design and operation. Electrochem. Soc. Interface. 2008;17(1):31-2.

[7] Wang $H$, Hao Q, Yang X, Lu L, Wang X. Graphene oxide doped polyaniline for supercapacitors. Electrochem. commun. 2009;11(6):1158-61.

[8] Zhao Q, Wangz X, Liu J, Wang H, Zhang Y, Gao $\mathrm{J}$, et al. Surface modification and performance enhancement of carbon derived from chromium carbide for supercapacitor applications. J. Electrochem. Soc. 2015;162(6):845-51.

[9] Kötz R, Carlen M. Principles and applications of electrochemical capacitors. Electrochim. 2010;45(15-16):2483-98.

[10] Zhang Y,Feng H, Wu X, Wang L, Zhang A, Xia $\mathrm{T}$, et al. Progress of electrochemical capacitor electrode materials: A review. Int. J. Hydrog. Energy. 2009;34(11):4889-99.

[11] Halama A, Szubzda B, Pasciak G. Carbon aerogels as electrode material for electrical double layer supercapacitors - Synthesis and properties. Electrochim. 2010;55(25):7501-5.

[12] Largeot C, Portet C, Chmiola J, Taberna PL, Gogotsi Y, Simon P. Relation between the ion size and pore size for an electric double-layer capacitor. J. Am. Chem. Soc.
2008;130(9):2730-1.

[13] Shi H. Activated carbons and double layer capacitance. Electrochim. 1996;41(10):1633-9.

[14]Frackowiak E, Béguin F. Carbon materials for the electrochemical storage of energy in capacitors. Carbon N. Y.2001;39(6):937-50.

[15]Pekala R. Organic aerogels from the polycondensation of resorcinol with formaldehyde. J. Mater. Sci.1989;24(9):3221-7.

[16] Czakkel O, Marthi K, Geissler E, László K. Influence of drying on the morphology of resorcinol-formaldehydebased carbon gels. Micropor. Mesopor. Mater.2005;86(1-3):124-33.

[17]Long D, Zhang J, Yang J, Hu Z, Cheng $\mathrm{G}$, Liu $\mathrm{X}$, et al. Chemical state of nitrogen in carbon aerogels issued from phenolmelamine-formaldehyde gels. Carbon N. Y. 2008;46(9):1259-62.

[18] Pekala R, Alviso C, Lu X, Gross J, Fricke $J$. New organic aerogels based upon a phenolic-furfural reaction. J. Non-Cryst Solids.1995;188(34-40):34-40.

[19]Long D, Zhang J, Yang J, Hu Z, Cheng G, Zhang R, et al. Preparation and microstructure control of carbon aerogels produced using $\mathrm{m}$-cresol mediated sol-gel polymerization of phenol and furfural. New Carbon Mater. 2008;23(2):165-70.

[20]Wu D, Fu R. Synthesis of organic and carbon aerogels from phenol-furfural by twostep polymerization. Micropor. Mesopor. Mater.2006;96(1-3):115-20.

[21] Scherdel C, Reichenauer G. Carbon xerogels synthesized via phenol-formaldehyde gels. Micropor. Mesopor. Mater. 2009;126(12):133-42.

[22] Fairén D, Carrasco F, Moreno C. Inter- and IntraPrimary-Particle Structure of Monolithic Carbon Aerogels Obtained with Varying Solvents. Langmuir. 2008;24(6):2820-5.

[23] Lin C, Ritter JA. Carbonization and activation of sol-gel derived carbon xerogels. Carbon. 2000;38(6):849-61.

[24] Hulicova D, et al. Effect of surface phosphorus functionalities of activated carbons containing oxygen and nitrogen on electrochemical capacitance. Carbon. 2009;47(6):1576-84.

[25] Park SJ, Jung WY. Effect of KOH activation on the formation of oxygen structure in activated carbons synthesized from polymeric precursor. J. Colloid Interface Sci. 2002;250(1):93-8.

[26] Carvalho A, Cardoso B, Pires J, De Carvalho 
MB. Preparation of activated carbons from cork waste by chemical activation with $\mathrm{KOH}$. Carbon. 2003;41(14):2873-6.

[27]Basta A, Fierro V, El-Saied H, Celzard A. 2-Steps $\mathrm{KOH}$ activation of rice straw: An efficient method for preparing highperformance activated carbons. Bioresour. Technol. 2009;100(17):3941-7.

[28] Lillo M, Cazorla D, Linares A. Understanding chemical reactions between carbons and $\mathrm{NaOH}$ and $\mathrm{KOH}$ an insight into the chemical activation mechanism. Carbon. 2003;41(2):267-75.

[29] Kierzek K, Frackowiak E, Lota G, Gryglewicz G, Machnikowski J. Electrochemical capacitors based on highly porous carbons prepared by $\mathrm{KOH}$ activation. Electrochim. 2004;49(4):515-23.

[30] Kıiliç M, Apaydın-Varol E, and Pütün AE. Preparation and surface characterization of activated carbons from Euphorbia rigida by chemical activation with $\mathrm{ZnCl} 2, \mathrm{~K} 2 \mathrm{CO} 3, \mathrm{NaOH}$ and H3PO4. Appl. Surf. Sci. 2012;261:247-54.

[31]Deng $H$, Li G, Yang $H$, Tang J, Tang J. Preparation of activated carbons from cotton stalk by microwave assisted $\mathrm{KOH}$ and $\mathrm{K} 2 \mathrm{CO} 3$ activation. Chem. Eng. J. 2010;163(3):373-81.

[32] Kim J, Lee D, Bae T, Lee Y. The electrochemical enzymatic glucose biosensor based on mesoporous carbon fibers activated by potassium carbonate. J. Ind. Eng. Chem. 2015;25:192-8.

[33] Mestre AS, Bexiga AS, Proença M, Andrade $M$, Pinto ML, Matos I, et al. Activated carbons from sisal waste by chemical activation with K2CO3: Kinetics of paracetamol and ibuprofen removal from aqueous solution. Bioresour. Technol. 2011;102(17):8253-60.

[34] Liu Y, Wang Y, Zhang G, Liu W, Wang D, Dong $Y$. Preparation of activated carbon from willow leaves and evaluation in electric double-layer capacitors. Mater. Lett. 2016;176:60-63.

[35]Zhang ZJ, Dong C, Ding XY, Xia YK. A generalized $\mathrm{ZnCl} 2$ activation method to produce nitrogen-containing nanoporous carbon materials for supercapacitor applications. J. Alloys Compd. 2015;636:275-81.

[36] Attia AA, Girgis BS, Fathy NA. Removal of methylene blue by carbons derived from peach stones by H3PO4 activation: Batch and column studies. Dye. Pigment. 2008;76(1):282-9.

[37] Gonzalez E, Cordero T, Rodriguez J, Cotoruelo $\mathrm{L}$, Rodriguez JJ. Removal of water pollutants with activated carbons prepared from $\mathrm{H} 3 \mathrm{PO} 4$ activation of lignin from kraft black liquors.
Water Res. 2004;38(13):3043-50.

[38] Kumar KS, AI Shoaibi A, Srinivasakannan C. A comparison of microstructure and adsorption characteristics of activated carbons by $\mathrm{CO} 2$ and $\mathrm{H} 3 \mathrm{PO} 4$ activation from date palm pits. New Carbon Mater. 2012;27(5):344-51.

[39] Pang L, Zou B, Han X, Cao L, Wang W, Guo Y. One-step synthesis of highperformance porous carbon from corn starch for supercapacitor. Mater. Lett. 2016;184:88-91.

[40]Pang L, Zou B, Zou Y,Han X, Cao L, Wang W, Guo $Y$ et al. A new route for the fabrication of corn starch-based porous carbon as electrochemical supercapacitor electrode material. Colloids Surfaces A: Physicochem. Eng. Asp. 2016;504:26-33.

[41] Wu M, Li L, Liu J, Li Y, Ai P, Wu W, et al. Templatefree preparation of mesoporous carbon from rice husks for use in supercapacitors. New Carbon Mater.2015;30(5):471-5.

[42] Qu J, Geng C, Lv S, Shao G, Ma S, Wu M. Nitrogen, oxygen and phosphorus decorated porous carbons derived from shrimp shells for supercapacitors. Electrochim. 2015;176:982-8.

[43] Molina M, Rodríguez F. Role of chemical activation in the development of carbon porosity. Colloids Surfaces A Physicochem. Eng. Asp. 2004;241(1-3):15-25.

[44] Sun J, Chen G, Wu J, Dong H, Xiong G. Bismuth vanadate hollow spheres: Bubble template synthesis and enhanced photocatalytic properties for photodegradation. Appl. Catal. B. 2013;132:304-14.

[45] Carrott PJM, Ribeiro MML. Lignin - from natural adsorbent to activated carbon: A review. Bioresour. Technol.2007; 98(12): 2301-12.

[46] Saha D, Li Y, Bi Z, Chen J, Keum JK, Hensley DK et al. Studies on Supercapacitor Electrode Material from Activated LigninDerived Mesoporous Carbon. Langmuir. 2014;30(3):900-10.

[47] Grishechko LI, Amaral G, Szczurek A, Fierro V, Kuznetsov BN, Celzard A. Lignin-phenolformaldehyde aerogels and cryogels. Microporous Mesoporous Mater. 2013;168:19-29.

[48] Castro CD, Quintana GC. Mixture Design Approach on the Physical Properties of LigninResorcinol-Formaldehyde Xerogels. Int. J. Polym. Sci.2015;2015.

[49]Dubinin M. Generalization of the theory of volume filling of micropores to nonhomogeneous microporous structures. Carbon. 1985;23(4):373-80. 
[50]Zapata Z, Carrasco F, Moreno C. Preparation, surface characteristics, and electrochemical doublelayer capacitance of $\mathrm{KOH}$-activated carbon aerogels and their $\mathrm{O}$ - and $\mathrm{N}$-doped derivatives. J. Power Sources. 2012;219:80-8.

[51] Rodríguez F, Linares A. Microporous structure of activated carbons as revealed adsorption methods. Chemistry and physics of carbon. 1989;21:1.

[52] Hayashi J., Kazehaya A, Muroyama K, Watkinson AP. Preparation of activated carbon from lignin by chemical activation. Carbon. 2000;38(13):1873-8.

[53] Chen F, Li J. Synthesis and Structural Characteristics of Organic Aerogels with Different Content of Lignin. Adv. Mater. Res. 2010;113:1837-40.

[54] Chen F, Xu M, Wang L, Li J. Preparation and characterization of organic aerogels from a lignin - resorcinol - formaldehíde copolymer. Bioresources. 2011;6(2):1262-72.

[55] Grishechkoa LI, Amaral G, SzczurekA, Fierro V, Kuznetsov BN, Pizzi A et al. New tannin-lignin aerogels. Ind. Crops Prod. 2013;41:347-55.

[56] Alonso MV, Oliet M, García J, Rodríguez F, Echeverría J. Gelation and isoconversional kinetic analysis of ligninphenol-formaldehyde resol resins cure. Chem. Eng. J. 2006;122(3):159-66.

[57] Li W, Wang J. Significant enhancement of the cycling performance and rate capability of the $\mathrm{P} / \mathrm{C}$ composite via chemical bonding ( $\mathrm{P}-\mathrm{C}$ ). J. Mater. Chem. A. 2016;4(2):505-11.

[58] Puziy AM, Poddubnaya OI, Ziatdinov AM. On the chemical structure of phosphorus compounds in phosphoric acidactivated carbon. Appl. Surf. Sci. 2006;252(23):8036-8.

[59] Liu Y, Li K, Liu Y, Pu L, Chen Z, Deng S. The high-performance and mechanism of $\mathrm{P}$-doped activated carbon as a catalyst for aircathode microbial fuel cells. J. Mater. Chem. A. 2015;3(42):21149-58.

[60] Qing X, Caoa Y, Wang J, Chen J, Lu Y. $\mathrm{P} / \mathrm{N} / \mathrm{O}$ co-doped carbonaceous materials based supercapacitor with voltage up to $1.9 \mathrm{~V}$ in the aqueous electrolyte. RSC Adv. 2014;4(99):55971-9.

[61]Xie H, Zhu Y, Wu Y, Wu Z, Liu E. The effect of hydroquinone as an electrolyte additive on electrochemical performance of the polyaniline supercapacitor. Mater. Res. Bull. 2014;50:3036.

[62] Mahata N, Pereira M, Suárez F, Martínez A, Tascón J, Figueiredo J. Tuning of texture and surface chemistry of carbon xerogels. J. Colloid Interface Sci. 2008;324(1-2):150-5.

[63] Su F, Poh CK, Chen JS, Xu G, Wang D, Li Q, et al. Nitrogen-containing microporous carbon nanospheres with improved capacitive properties. Energy Environ. Sci. 2011;4(3):717-24.

[64] Raymundo E, Béguin F. Application of nanotextured carbons for supercapacitors and hydrogen storage. In: Interface Science and Technology. Elsevier; 2006. p. 293-343.

[65]Bhattacharjya D, Yu JS. Activated carbon made from cow dung as electrode material for electrochemical double layer capacitor. J. Power Sources. 2014;262:224-231.

[66] Isil I, Inal G, Holmes SM, Banford A, Aktas Z. The performance of supercapacitor electrodes developed from chemically activated carbon produced from waste tea. Appl. Surf. Sci. 2015; 357:696-703.

[67]Liu X, Zhang R, Zhang L, Long D, Qiao $\mathrm{W}$, Yang $\mathrm{J}$ et al. Impedance of carbon aerogel/ activated carbon composites as electrodes of electrochemical capacitors in aprotic electrolyte. New Carbon Mater. 2007;22(2):153-8.

[68] Bordjiba T, Mohamedi M, Dao LH. Synthesis and electrochemical capacitance of binderless nanocomposite electrodes formed by dispersion of carbon nanotubes and carbon aerogels. J. Power Sources. 2007;172(2):991-8

[69] Qu D, Shi H. Studies of activated carbons used in double-layer capacitors. J. Power Sources.1998;74(1):99-107. 